\title{
Treatment of Unstable Intertrochanteric Fractures in Elderly Patients Using a New Technique of Wire Tension Band Ventral Compression Wiring Combined With Artificial Femoral Head Replacement
}

\section{Jiajing Ye}

WenZhou Medical College Affiliated Taizhou Hospital: Taizhou Hospital of Zhejiang Province

\section{Zhong Zhu}

WenZhou Medical College Affiliated Taizhou Hospital: Taizhou Hospital of Zhejiang Province Lingjun Jiang

WenZhou Medical College Affiliated Taizhou Hospital: Taizhou Hospital of Zhejiang Province Haizhao Wu

WenZhou Medical College Affiliated Taizhou Hospital: Taizhou Hospital of Zhejiang Province Shouli Wang

WenZhou Medical College Affiliated Taizhou Hospital: Taizhou Hospital of Zhejiang Province Qiaohong Pan

WenZhou Medical College Affiliated Taizhou Hospital: Taizhou Hospital of Zhejiang Province Zhongyi Chen ( $\sim$ czy6865@163.com)

Taizhou Hospital of Zhejiang Province affiliated to Wenzhou Medical University

\section{Research article}

Keywords: intertrochanteric fracture, femoral head replacement, steel wire, wiring technology, ventral compression

Posted Date: August 31st, 2021

DOI: https://doi.org/10.21203/rs.3.rs-847260/v1

License: (c) (1) This work is licensed under a Creative Commons Attribution 4.0 International License. Read Full License 


\section{Abstract}

Background: This study evaluates the effects of wire tension band ventral compression wiring technology paired with artificial femoral head replacement according to the different types of intertrochanteric fractures of the greater trochanter in elderly patients.

Methods: Thirty-eight patients with unstable intertrochanteric fractures of the femur treated with artificial femoral head replacement between January 2015 and August 2019 were included. According to the fracture line of the greater trochanter, a new classification system was proposed. Type A fractures include transverse fractures from the greater trochanter tip to the base (2 patients). Type B fractures include oblique fractures from the greater trochanter tip to the base (according to the fracture line direction, type B was further divided into types B1 [4 patients], and B2 [24 patients]). the fracture line of type C fractures runs from the greater trochanter to near the femur end (8 patients). Different wire tension belt ventral compression wiring technologies were used for each fracture type. The Harris hip function score, Parker activity score, and hip pain were evaluated during the follow-up period. Fracture healing and prosthesis positioning, loosening, and dislocation were evaluated using radiographs.

Results: The average follow-up period was $28.6 \pm 5.8$ months. Deep vein embolism was noted in one patient, heterotopic ossification in another, and steel wire fractures in another. All patients had satisfactory fracture healing and femoral prosthesis positioning and no chronic pain. The mean Harris hip function score was $7.21 \pm 2.58$ preoperatively and $84.74 \pm 3.82$ at the final follow-up $(F=-48.13, P<$ $0.001)$.

Conclusion: The use of different wire tension band ventral compression wiring technology based on different types of femoral rotation fractures combined with artificial femoral head replacement in elderly patients with unstable intertrochanteric fractures results in favorable clinical outcomes.

\section{Background}

Femoral intertrochanteric fractures are common in elderly patients, accounting for approximately 31$51 \%$ of hip fractures [1]. As the population ages, the incidence of femoral intertrochanteric fractures will further increase. Most femoral intertrochanteric fractures in elderly patients are comminuted and occur in patients with comorbidities such as osteoporosis. According to previous studies, the mortality rate of femoral intertrochanteric fractures in elderly patients is as high as $27-30 \%[2,3]$. A reasonable and effective treatment that allows patients to get out of bed early, reduces postoperative complications, and reduces the risk of death is the focus of current research [4]. Surgical treatment has become the standard of care, and there are two surgical treatments used for intertrochanteric fractures: internal fixation and joint replacement. Internal fixation for comminuted intertrochanteric fractures is associated with several complications, including fixation failure and fracture nonunion [5]. Therefore, hip replacement surgery is the first choice of treatment for elderly patients with osteoporosis and unstable intertrochanteric fractures [6]. However, the fixation of the greater trochanter is challenging during hip replacement surgery in these 
patients. This study retrospectively analyzed the main points of operation and the clinical efficacy of the combination of wire tension band ventral compression wiring technology with artificial femoral head replacement using a new classification system to determine which wiring technology should be used in each patient.

\section{Methods}

\section{Patients}

This study included 43 patients aged $\geq 70$ years with closed femoral intertrochanteric fractures who underwent hip replacement surgery with an artificial femoral head using a new type of steel wire tension band ventral compression wiring technique at our institution between January 2015 and August 2019. Patients who had surgery within two weeks of the injury, were classified as type III or V according to the Evans-Jensen classification system, and were followed for at least 24 months postoperatively were included. Patients with concomitant fractures, significantly abnormal blood coagulation functioning, incomplete clinical data, short follow-up periods (<24 months), or contraindications to surgery were excluded from the study.

This study was approved by the Ethics Committee of Taizhou Hospital in Zhejiang Province (approval number: K20191206), and all patients provided informed consent. The study was conducted in accordance with the principles of the Declaration of Helsinki.

\section{Surgical methods}

One senior physician and his team conducted the surgery for each patient included in this study. The patient was positioned on his or her contralateral side and a subarachnoid block was combined with epidural anesthesia or general anesthesia. A posterolateral approach was used. The skin, subcutaneous tissue, and fascia lata were sequentially incised, and the front and back edges of the fascia were separated bluntly to reveal the short external rotator group and the posterior edge of the gluteus medius muscle. The short external rotator group was detached at the femoral attachment and used to protect the sciatic nerve. The upper femur was exposed by separating the subperiosteal muscles, allowing the determination of the displacement of the intertrochanteric fracture. The sac was exposed via a conventional femoral neck osteotomy, and the diameter of the femoral head was measured, the greater trochanter fracture piece was pulled forward, the direction of the medullary cavity was determined, and the femoral medullary cavity was reamed to select the appropriate femoral stem prosthesis. The prosthesis was placed into the medullary cavity along the normal anteversion angle. The trial model of the femoral head was installed, and the length of the lower limbs and the stability of the hip joint were determined prior to the installation of the prosthesis. According to the displacement of the large and small trochanter fracture pieces, the fracture pieces were circulated and fixed around the prosthesis using steel wires, as described below. Bone cement was used for fixation in patients with osteoporosis based on the preoperative bone mineral density score. Biological fixation was used in patients without osteoporosis. The fascia lata was continuously sutured, and the incision was closed. 


\section{Steel wire tension band ventral compression fixation model and wire tying method according to the new classification system}

A novel fracture classification system for greater trochanter fractures of femoral intertrochanteric fractures according to the location of the fracture line was created to achieve better fracture fixation. The fractures were divided into three types: $A, B$, and $C$ according to the location of the fracture line (Fig. 1). Type A fractures have transverse fracture lines from the tip of the greater trochanter to the level of the piriform fossa (the fracture may be in one or more pieces). Type A fractures are typically fixed with two steel wires (Fig. 2). Type B fractures have oblique fracture lines from the tip of the greater trochanter to the base of the greater trochanter above the level of the lesser trochanter. This type is further classified according to the direction of the fracture line: type B1 fractures run obliquely anteriorly and distally from behind the greater trochanter (Fig. 3), and type B2 fractures run obliquely posteriorly and distally from the upper anterior to the lower posterior (Fig. 4). The greater trochanter fracture may be in one or more pieces, and type $B$ fractures are typically fixed with two or three steel wires. Type $C$ fracture lines extend from the greater trochanter to the lower trochanter plane, and the fracture may be in one or more pieces. This type is fixed with three to five steel wires according to the extent of fracture involvement and the degree of comminution (Fig. 5). To facilitate fixation, the steel wire perforates the tip of the greater trochanter. The actual trochanter tip has tough soft tissue that the steel wire can be directly inserted underneath.

As the main abductor muscle, the gluteus medius stops at the greater trochanter and is very important for maintaining balance. Stress after a greater trochanter fracture occurs in an upward and ventral direction and can be mainly attributed to the gluteus medius [7]. The fracture should be fixed according to the direction and position of the fracture line and the direction of the tension of the gluteus medius muscle. Fixation with compression of the steel wire of the ventral ring should also be considered.

\section{Postoperative treatment and follow-up methods}

After the operation, antibiotic, prophylactic, anti-infection, and anticoagulation therapies were administered to all patients. The white blood cell count, erythrocyte sedimentation rate, and C-reactive protein were monitored to evaluate the healing of the surgical incision. Patients were permitted to get out of bed with the assistance of a walker and perform some weight-bearing activities on postoperative day 1. The patients gradually returned to full weight-bearing and performed various hip joint function exercises during postoperative rehabilitation. Outpatient visits were conducted at one, three, six, and 12 months postoperatively. After that time, the follow-up visits occurred annually. During the follow-up period, anterior radiographs of the pelvis and anteroposterior and lateral radiographs of the middle and upper segments of the affected femur were obtained. The Harris hip function score (HHS), Parker activity score [8], and pain score (visual analogue score (VAS)) were used to evaluate the function of the hip joint. Fracture healing, the condition of the steel wire, and the position of the prosthesis were evaluated via radiographs. Alendronate was administered postoperatively to prevent osteoporosis.

\section{Statistical methods}


All statistical analyses were performed using SPSS version 22.0 statistical software (IBM, New York, United States). Continuous data are expressed as mean \pm standard deviation. The HHS, Parker activity score, and pain score were analyzed via a one-way analysis of variance and compared using a paired ttest. Statistical significance was set at $p<0.05$.

\section{Results}

\section{Patient characteristics}

Of the 43 patients enrolled in this study, two died early during the follow-up period and three were lost to follow-up. The remaining 38 patients consisted of 22 males and 16 females with a mean age of 84.8 years (range: 75-95 years). Twenty-eight patients had fractures of the left hip. Twenty-three patients had Evans-Jensen type III fractures, while 15 had type $V$ fractures. The mean bone mineral density $T$ value was $-2.95 \pm 0.32$. Thirty-two patients had osteoporosis, 30 had primary hypertension, 16 had diabetes, 10 had liver insufficiency, eight had chronic bronchitis, five had coronary atherosclerotic heart disease, and five had renal insufficiency.

According to the novel classification system, two patients had type A fractures, four had type B1 fractures, 24 had type $B 2$ fractures, and eight had type $C$ fractures. Patients with type $A$ fractures were fixed with two steel wires, whereas two or three wires were used in patients with type B fractures, and three to five wires were used in patients with type $C$ fractures. Bone cement was also used for fixation in the 32 patients with osteoporosis, while biological fixation was permitted in the remaining six patients. The mean intraoperative blood loss was $238.42 \pm 74.01 \mathrm{~mL}$ (range: $150-380 \mathrm{~mL}$ ), and the mean operation time was $84.74 \pm 10.80$ minutes (range: $65-120$ minutes). The mean hospital stay was $9.50 \pm$ 1.64 days (range: 6-11 days). Autologous blood transfusions were performed during the operation and one patient received a foreign body. The erythrocyte suspension was $2 \mathrm{U}$, and all surgical incisions healed by first intention.

\section{HHS}

The mean HHS was $7.21 \pm 2.58$ points preoperatively, $64.89 \pm 4.14$ points three months postoperatively, and $84.74 \pm 3.82$ points two years postoperatively. The HHSs at three months and two years postoperatively were significantly different $(P<0.05$, Table 1$)$. 
Table 1

HHSs

\begin{tabular}{|c|c|c|c|c|c|}
\hline & Pain score & $\begin{array}{l}\text { Function } \\
\text { score }\end{array}$ & $\begin{array}{l}\text { Deformity } \\
\text { score }\end{array}$ & $\begin{array}{l}\text { Range of } \\
\text { motion }\end{array}$ & Total \\
\hline Preoperative & $\begin{array}{l}5.50 \pm \\
2.15^{\star} \#\end{array}$ & $0 * \#$ & $\begin{array}{l}0.868 \pm \\
0.704^{\star} \#\end{array}$ & $\begin{array}{l}0.84 \pm \\
0.68^{\star} \#\end{array}$ & $\begin{array}{l}7.21 \pm \\
2.58^{\star} \#\end{array}$ \\
\hline $\begin{array}{l}\text { Three months } \\
\text { postoperatively }\end{array}$ & $\begin{array}{l}33.92 \pm \\
2.02 * \&\end{array}$ & $\begin{array}{l}25.01 \pm \\
3.17 * \&\end{array}$ & $\begin{array}{l}2.868 \pm \\
0.66 * \&\end{array}$ & $\begin{array}{l}3.11 \pm \\
0.76 * \&\end{array}$ & $\begin{array}{l}64.89 \pm \\
4.14 * \&\end{array}$ \\
\hline $\begin{array}{l}\text { Two years } \\
\text { postoperatively }\end{array}$ & $\begin{array}{l}40.24 \pm \\
2.61 \# \&\end{array}$ & $\begin{array}{l}36.47 \pm \\
2.87 \# \&\end{array}$ & $\begin{array}{l}4.026 \pm \\
0.788 \# \&\end{array}$ & $\begin{array}{l}4.01 \pm \\
0.74 \# \&\end{array}$ & $\begin{array}{l}84.74 \pm \\
3.82 \# \&\end{array}$ \\
\hline F score & 25.11 & 21.65 & 18.67 & 19.05 & 48.13 \\
\hline$P$-value & $<0.001$ & $<0.001$ & $<0.001$ & $<0.001$ & $<0.001$ \\
\hline \multicolumn{6}{|c|}{ Data are presented as mean \pm standard deviation. } \\
\hline
\end{tabular}

\section{Parker activity score}

The mean Parker activity score was 0 points preoperatively, $6.01 \pm 0.98$ points three months postoperatively, and $7.92 \pm 0.75$ points two years postoperatively. The preoperative and 2-year postoperative Parker activity scores were significantly different $(P<0.05$, Fig. 6$)$.

\section{Pain score}

The mean VAS score for the hip joint was $6.34 \pm 0.91$ points preoperatively, $2.66 \pm 0.85$ points three months postoperatively, and $1.29 \pm 0.65$ points two years postoperatively. The preoperative VAS scores were significantly different than those at three months and two years postoperatively (both $P<0.05$, Fig. 7).

\section{Time until initial weight-bearing activity}

The intraoperative examination revealed a firm fracture fixation in all patients with no displacement of the fractured end of the movable hip joint. Twenty-five patients were able to walk on the first postoperative day, 10 walked on postoperative day 2, and two walked on postoperative day 3 . One patient had repeated dizziness and discomfort and did not stand or walk until postoperative day 7.

\section{Fracture healing and complications}

The average follow-up period was $28.6 \pm 5.8$ months (range: $24-38$ months). Postoperative deep vein thrombosis occurred in one patient who recovered after anticoagulation treatment. One patient developed heterotopic ossification and experienced mild pain and discomfort that was treated with oral non- 
steroidal anti-inflammatory analgesics. Another patient experienced wire breakage 12 months postoperatively. In all patients, the fracture was healed at the last follow-up visit. The mean fracture healing time was $9.50 \pm 1.64$ months (range: 7-113 months). There was no loosening or dislocation of the prosthesis and no dislocation of the greater trochanter at the last follow-up. No patient reported chronic pain.

\section{Typical case 1}

A 76-year-old female with a history of osteoporosis developed pain, deformity, and limited mobility of the left hip 1 day after trauma. An anterior pelvic radiograph indicated a left intertrochanteric fracture (Evans-

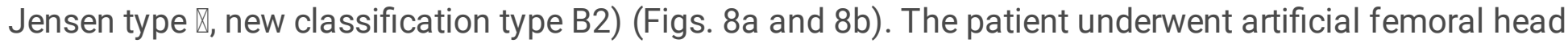
replacement with steel wire fixation of the comminuted intertrochanteric fracture of the left femur. At the final follow-up visit, the patient's hip was active with no pain or obvious claudication. The final radiograph revealed a well-healed fracture with no loosening of the prosthesis (Fig. 8).

\section{Typical case 2}

An 86-year-old female with a history of osteoporosis had pain, deformity, and limited mobility of the right hip for two days after trauma. An anterior pelvic radiograph suggested a right comminuted intertrochanteric fracture of the right femur (Evans-Jensen type $\mathrm{V}$, new classification type $\mathrm{C}$ ). The patient underwent artificial femoral head replacement with steel wire internal fixation of the intertrochanteric fracture of the right femur (Fig. 9).

\section{Discussion}

Due to the mechanical characteristics of the fracture site, improper selection of internal fixation, concomitant osteoporosis, and other factors, the incidence of postoperative internal fixation failure is approximately $4-20 \%$ in elderly patients with comminuted femoral intertrochanteric fractures $[9,10]$. Therefore, arthroplasty has been recognized as an acceptable treatment for these patients [11], including the use of cemented prostheses [12] and biological fixation methods [13]. Hip prostheses result in favorable clinical outcomes, allowing for patients to get out of bed early, reducing postoperative complications, and improving the patients' quality of life. However, fixation of the greater trochanter during hip joint replacement surgery is challenging. This study presents an effective method for the treatment of unstable intertrochanteric fractures in the elderly.

\section{A novel classification system for greater trochanter fractures}

A novel classification system for greater trochanter fractures, based on the Evans-Jensen types III and V fractures has been introduced in this study. The specific fracture and displacement of the greater trochanter fracture in the Evans-Jensen classification system has not been analyzed thoroughly. The new classification system is mainly based on the displacement direction and location of the greater trochanter fracture line. This new system aims to result in better fixation of greater trochanter fractures. Among the 
38 patients in this study, two had type A fractures, four had type B1 fractures, 24 had type B2 fractures, and eight had type $C$ fractures. Overall, 18 patients had simple fractures of the greater trochanter (twopart fractures) and 20 patients had comminuted fractures ( $\geq$ three-part fractures). Several muscles, including the gluteus medius and gluteus minimus, attach to the greater trochanter. The main pulling direction of the gluteus medius is upward and backward; therefore, the greater trochanter will be displaced in this direction if fractured. This anatomy accounts for the high proportion of patients with type B2 fractures in this study. The direction of intraoperative wire binding and compression must be determined based on the direction of the displacement of the greater trochanter, and the direction of intraoperative wire compression should be the opposite of the direction of the bone block pulling to resist the force of fracture displacement. In this study, the ventral compression fixation achieved using steel wire tension bands based on the new classification system resulted in favorable fixations.

\section{Clinical efficacy of steel wire tension bands according to the new classification system}

Several fixation methods are currently used to treat greater trochanter fractures, though these methods are complicated by fixation failure, osteolysis, and prosthesis loosening, which is a common cause of hip revision surgery [14]. Jeffrey et al. [15] reported the use of a claw plate to fix greater trochanter fractures during hip arthroplasty. Three of the 31 patients in their study developed greater trochanter bursitis with obvious pain in the lateral hip that improved after the removal of the plate. Three patients in the previous study experienced bone nonunion and one patient had an infection. No patients in the current study reported lateral hip pain, which may be due to the fact that the steel wire and the steel plate are relatively small, resulting in less irritation to the soft tissue. The large claw plate likely results in squeezing of the bursa and friction, leading to bursitis. An in vitro model was used to determine the usefulness of a cable binding system to fix greater tuberosity fractures in a previous study [16]. This system has a stronger biomechanical structure; however, contact between the rough cable and the femoral stem resulted in metal particles that caused prosthesis loosening. In this study, a steel wire tension band was used to fix the steel wire. The steel wire is relatively smooth and is not associated with complications of metal particles. Another study including three patients with greater trochanter fractures reported the use of a steel plate combined with a steel cable to fix the greater tuberosity [17]. In this previous study, all three patients had loosening and displacement of the steel cable, resulting in fixation failure. The fixation failure was attributed to the fixed steel cable and a problem with the pressurizing device. The pressurization of the steel wire is dependent on the twisting and pressurization between the two ends of the steel wire, which is simple, convenient, and intuitive. Takahira et al. [18] used steel cables and steel plates to fix greater trochanter fractures during hip arthroplasty and reported complications including fractures of the steel cables and bone resorption around the steel cables. Compressed steel cables are stronger than steel wires or steel cables. As joint replacement surgeries are typically conducted in elderly patients, the use of steel cable may lead to accidental fractures during compression. In this study, the Harris hip function score, Parker activity score, and pain score were significantly improved during the follow-up period. The greater trochanter fracture healed well, and only one patient had wire breakage. There were no common complications including greater trochanter bursitis, osteolysis caused by metal wear, or prosthesis loosening. 


\section{Ventral compression fixation with steel wire tension band}

Steel wire tying is essential for ventral compression fixation with steel wire tension bands. Tension band fixation technology has achieved satisfactory results as a standard internal fixation method for patella and olecranon fractures, which are similar to greater trochanter fractures $[19,20]$. The compression and stabilization technology of the ventral fracture surface in the band fixation technique is important. If there is no ventral compression, the failure rate of internal fixation is greatly increased. However, ventral compression has previously been ignored in the banding technology of greater trochanter fractures [21, 22]. The fixation effect achieved using this technology is reliable, even when the greater trochanter fracture is comminuted. As the greater trochanter is attached to the tough muscle fascia, steel wire can be used during fixation. Banding fixation can be used to suture comminuted fracture fragments and preserve the blood supply of the fracture fragments, which aids in fracture healing.

For type A fractures, a ring-shaped steel wire and a figure eight-shaped steel wire are used for fixation. The ring-shaped steel wire is wound from the tip of the greater trochanter to the lower part of the lesser trochanter and is fixed on the ventral side posterolaterally from the greater trochanter. The figure eightshaped steel wire surrounds the fractured portion. For type B fractures, two steel wires are used during fixation. The ring-shaped steel wire fixation is the same as that for type $A$ fractures, while the direction of the figure eight-shaped steel wire is dependent on the direction of the fracture line. Type B1 fractures are fixed in the posterolateral direction of the greater trochanter, and type B2 fractures are fixed in the anterolateral direction of the rotor. For type $\mathrm{C}$ fractures, three or more steel wires are generally used for fixation. The first ring steel wire is the same as that for type A fractures, the second ring steel wire is wound below the lesser trochanter perpendicular to the fracture line for ring fixation, and the third figure eight-shaped steel wire achieves compression fixation on the opposite side of the fracture line. When the fracture line is inferior to the lesser trochanter, one or more steel wires can be added below the lesser trochanter for ring fixation.

\section{Limitations}

This study is not without limitations. It is a retrospective study that includes a small number of patients and a relatively short follow-up period, which may affect the reliability of the data. In addition, no control group was included. Therefore, a large-scale, long-term follow-up study that includes a control group is needed.

\section{Conclusions}

The results of this study indicate that the novel classification system for greater trochanter fractures based on the wire tension band ventral compression wiring technology combined with artificial femoral head replacement achieves favorable clinical outcomes in elderly patients with comminuted femoral intertrochanteric fractures. 


\section{Abbreviations}

HHS: Harris hip function score; VAS: visual analogue score

\section{Declarations}

\section{Ethics approval and consent to participate:}

This study was approved by the Ethics Committee of Taizhou Hospital in Zhejiang Province (approval number: K20191206) and all the participants provided informed consent.

\section{Consent for publication:}

Written informed consent for publication was obtained from all participants

\section{Competing interests:}

The authors declare that they have no competing interests

\section{Availability of data and materials:}

The datasets used or analyzed during the current study are available from the corresponding author on reasonable request.

\section{Competing interests:}

None

\section{Funding:}

This study was supported by funding from the Zhejiang Province Medical Science and Technology Project (grant numbers 2020KY1033 and 2019RC307).

\section{Authors' contributions:}

YJJ contributed significantly to the analyses and wrote the manuscript. CZY designed the study. WHZ and WSL performed the data analysis. ZZ, JLJ, and PQH assisted analysis with constructive discussions. All authors read and approved the final manuscript.

\section{Acknowledgements:}

None

\section{References}


1. Haidukewych GJ, Israel TA, Berry DJ. Reverse obliquity fractures of the intertrochanteric region of the femur. J Bone Joint Surg Am. 2001;83:643-50. DOI:10.2106/00004623-200105000-00001

2. Chehade MJ, Carbone T, Awwad D, Taylor A, Wildenauer C, Ramasamy B, et al. Influence of fracture stability on early patient mortality and reoperation after pertrochanteric and intertrochanteric hip fractures. J Orthop Trauma. 2015;29:538-43. DOI:10.1097/BOT.0000000000000359

3. Forster MC, Calthorpe D. Mortality following surgery for proximal femoral fractures in centenarians. Injury. 2000;31:537-9. DOI:10.1016/S0020-1383(00)00049-8

4. Tang PF. Progress and strategies in treatment of intertrochanteric fractures. Chin J Orthop Trauma. 2017;19:93-4. DOI:10.3760/cma.j.issn.1671-7600.2017.02.001

5. Wang SD, Dong QR, Xu YJ. Influencing factors related to failed internal fixation of osteoporotic femoral intertrochanteric fractures in elderly patients. Chin J Orthop Trauma. 2014;16:656-61. DOI:10.3760/cma.j.issn.1671-7600.2014.08.003

6. Abdelkhalek M, Ali AM, Abdelwahab M. Cemented bipolar hemiarthroplasty with a cerclage cable technique for unstable intertrochanteric hip fractures in elderly patients. Eur J Orthop Surg Traumatol. 2013;23:443-8. DOI:10.1007/s00590-012-1006-z

7. Anagnostakos PK, Kohn D, Lorbach O. Osteotomy of the greater trochanter. Orthopade. 2013;42:32231. DOI:10.1007/s00132-012-2013-9

8. Parker MJ, Palmer CR. A new mobility score for predicting mortality after hip fracture. J Bone Joint Surg Br. 1993;75:797-8囚 DOI:10.1302/0301-620X.75B5.8376443

9. Liu Y, Sun Y, Fan L, Hao J. Perioperative factors associated with hidden blood loss in intertrochanteric fracture patients. Musculoskelet Surg. 2017;101:139-44. DOI:10.1007/s12306-0160447-7

10. Niu E, Yang A, Harris AHS, Bishop J. Which fixation device is preferred for surgical treatment of intertrochanteric hip fractures in the United States? A survey of orthopaedic surgeons. Clin Orthop Relat Res. 2015;473:3647-55. DOI:10.1007/s11999-015-4469-5

11. Li Y, Qi SUN, Yi Li-ming. Comparison of intramedullary fixation and hemiarthroplasty for treatment of femoral intertrochanteric fracture in the elderly patients following stroke. Chin J Orthop Trauma. 2013;15:397-401. DOI:10.3760/cma.j.issn.1671-7600.2013.05.007

12. Wang BY, Zhao JN, Zhou LW. Cemented femoral head replacement for treatment of femoral intertrochanteric fractures in the elderly. Chin J Orthop Trauma. 2009;11:187-8. DOI:10.3760/cma.j.issn.1671-7600.2009.02.022

13. Wang YH, Zhou SP, Chen B, Wang A. Treatment of comminuted intertrochanteric fractures with uncemented long-stem hemiarthroplasty in elderly patients. Chin J Orthop Trauma. 2018;20:267-70. DOI:10.3760/cma.j.issn.1671-7600.2018.03.016

14. He B, Zhang MF, Sheng Y, Zhao X, Ying Z, Wei W, et al. Evaluation of causes and clinical effects of revision surgery after artificial hip replacement. Chin J Orthop. 2019;39:909-917. DOI: $10.3760 /$ cma.j.issn.0253ख2352.2019.15.001 
15. Zarin JS, MD, Zurakowski D, Burke DW. Claw plate fixation of the greater trochanter in revision total hip arthroplasty[J]. J Arthroplasty. 2009;24:272-80. DOI:10.1016/j.arth.2007.09.016

16. Thakur NA, Crisco JJ, Moore DC, Froehlich JA, Limbird RS, Bliss JM. An improved method for cable grip fixation of the greater trochanter after trochanteric slide osteotomy. J Arthroplasty. 2010;25:31924. DOI:10.1016/j.arth.2008.10.006

17. Klinge SA, MD, Vopat BG, Daniels AH, Bariteau JT, Rubin LE, Limbird R. Early catastrophic failure of trochanteric fixation with the Dall-Miles Cable Grip system. J Arthroplasty. 2014;29:1289-91.

DOI:10.1016/j.arth.2014.01.001

18. Takahira N, Itoman M, Uchiyama K, Takasaki S, Fukushima K. Reattachment of the greater trochanter in total hip arthroplasty: the pin-sleeve system compared with the Dall-Miles cable grip system. Int Orthop. 2010;34:793-7. DOI:10.1007/s00264-010-0989-5

19. Lotke PA, Ecker ML. Transverse fractures of the patella. Clin Orthop Relat Res. 1981;158:180-4. DOI:10.1097/00003086-198107000-00026

20. Wang CX, Tan L, Qi BC, Hou X-F, Huang Y-L, Zhang H-P, et al. A retrospective comparison of the modified tension band technique and the parallel titanium cannulated lag screw technique in transverse patella fracture. Chin J Traumatol. 2014;17:208-13. DOI:10.3760/cma.j.issn.10081275.2014.04.005

21. Archibeck MJ, Rosenberg AG, Berger RA, Silverton CD. Trochanteric osteotomy and fixation during total hip arthroplasty. J Am Acad Orthop Surg. 2003;11:163-73. DOI:10.5435/00124635-20030500000003

22. Dall DM, Miles AW. Re-attachment of the greater trochanter. The use of the trochanter cable-grip system. J Bone Joint Surg Br. 1983;65:55-9. DOI:10.1302/0301-620X.65B1.6337168

\section{Figures}
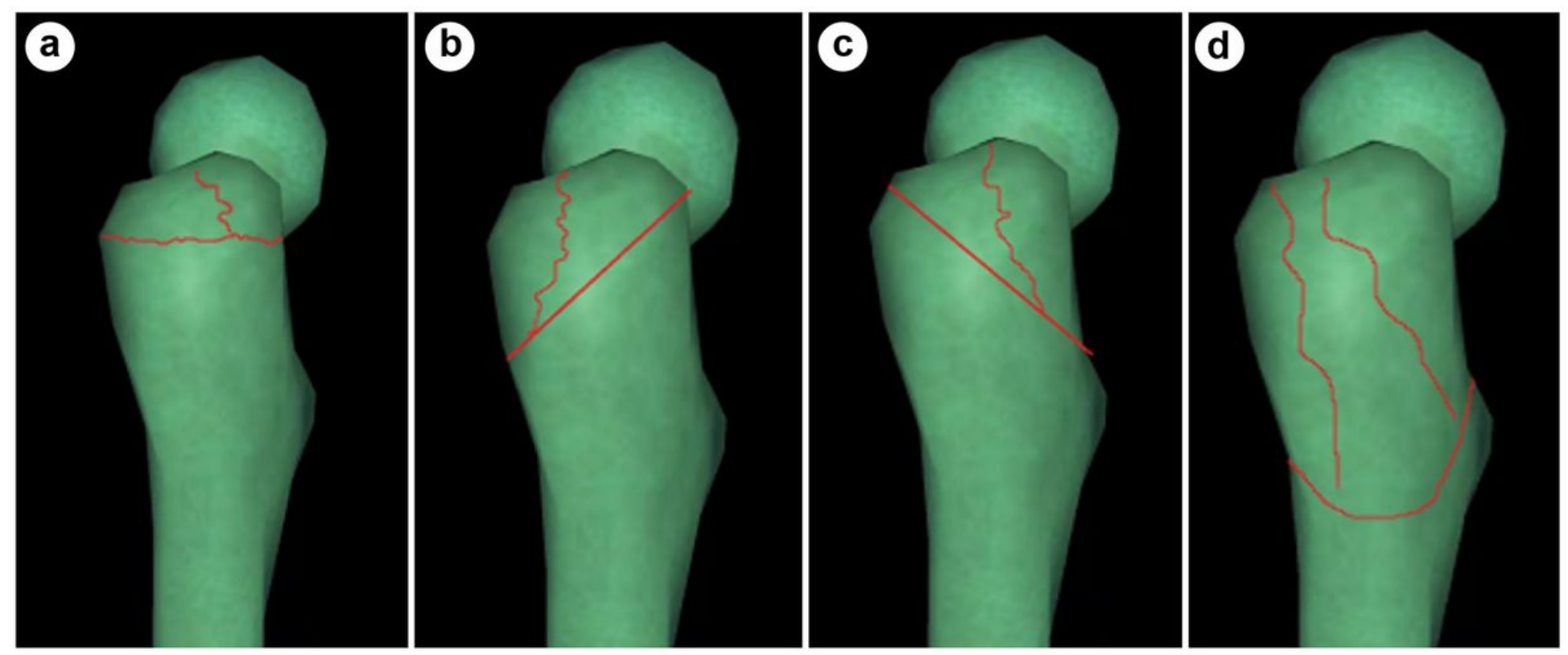


\section{Figure 1}

Initial classification of femoral greater trochanter fracture a: Type A fractures have transverse fracture lines from the tip of the greater trochanter to the level of the piriform fossa. b. Type B1 fractures have oblique fracture lines from the tip of the greater trochanter to the base of the greater trochanter above the level of the lesser trochanter. c. Type B2 fractures have oblique fracture lines from the tip of the greater trochanter to the base of the greater trochanter above the level of the lesser trochanter. The fracture line runs obliquely from the upper anterior to the lower posterior. $d$. Type $C$ fractures have fracture lines extending from the greater trochanter to the lower trochanter plane.

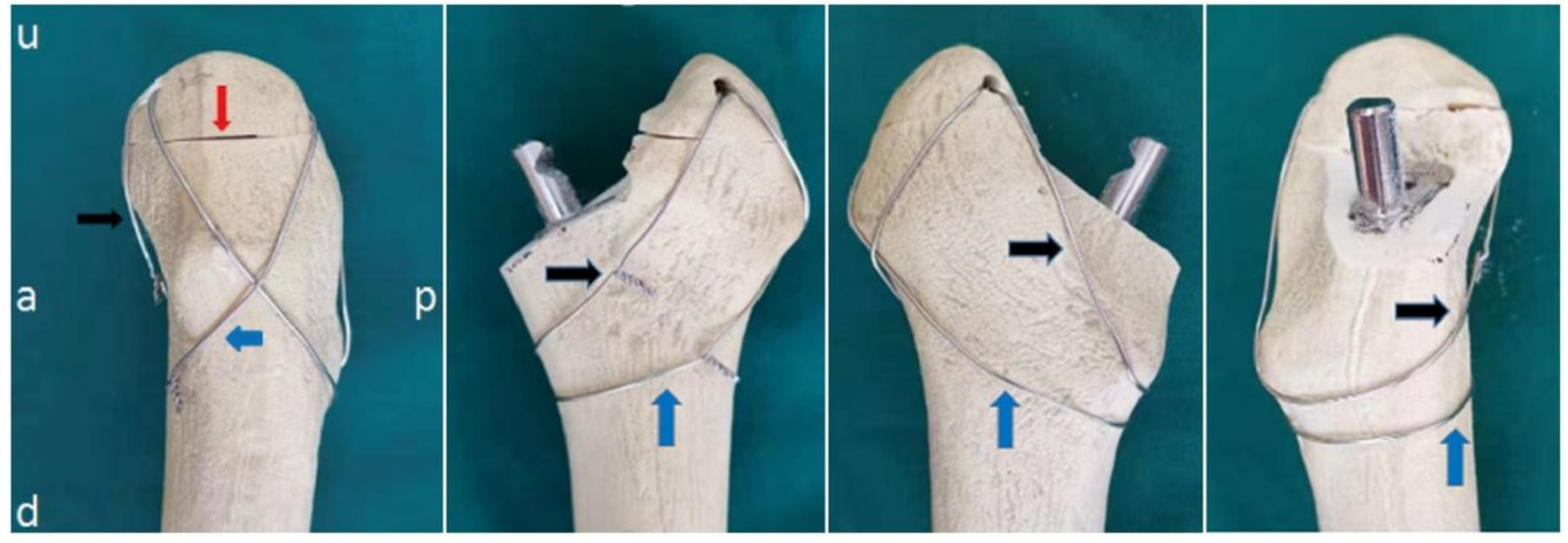

Figure 2

Type A fractures Type A fractures are fixed with a steel wire tension band. A steel wire (blue arrow) penetrates the greater trochanter and provides fixation under the lesser trochanter in a figure eight shape. A second steel wire (black arrow) also penetrates the greater trochanter. The hole is wound under the lesser trochanter for ring fixation as a fixed wire for ventral compression. The red arrow represents the fracture line., with $u, d, a$, and $p$ denoting the upward, downward, anterior, and posterior anatomic directions, respectively. The steel wire is $2 \mathrm{~mm}$ in diameter (Resorba medical $\mathrm{GmbH}$, Germany).

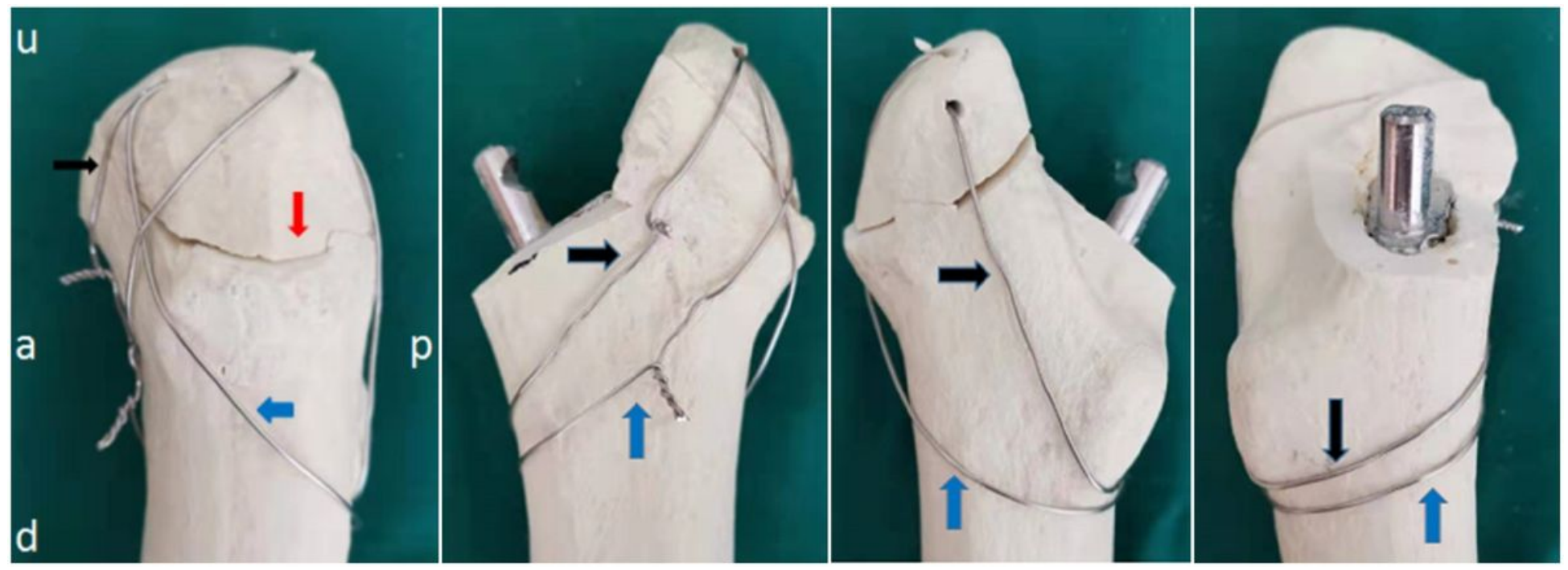




\section{Figure 3}

Type B1 fractures Oblique fractures from the tip of the greater trochanter to the base are classified as type B1 fractures. The fracture line runs from the tip of the greater trochanter to the base on the side of the lesser trochanter. The red arrow represents the fracture line. A steel wire (blue arrow) perforates the greater trochanter and is shaped in a figure eight to fix the greater trochanter to the lesser trochanter. The opposite side is tightened, and a second steel wire (black arrow) also penetrates the tip of the rotor and is fixed in a ring on the opposite side of the lesser trochanter. The wire is tightened to provide ventral compression. The wire is positioned in front of the greater trochanter fracture line, and both wires are wound around the trochanter to fix it under the rotor, with $\mathrm{u}, \mathrm{d}$, a, and p denoting upward, downward, anterior, and posterior anatomic directions, respectively. The steel wire is $2 \mathrm{~mm}$ in diameter (Resorba medical GmbH, Germany).

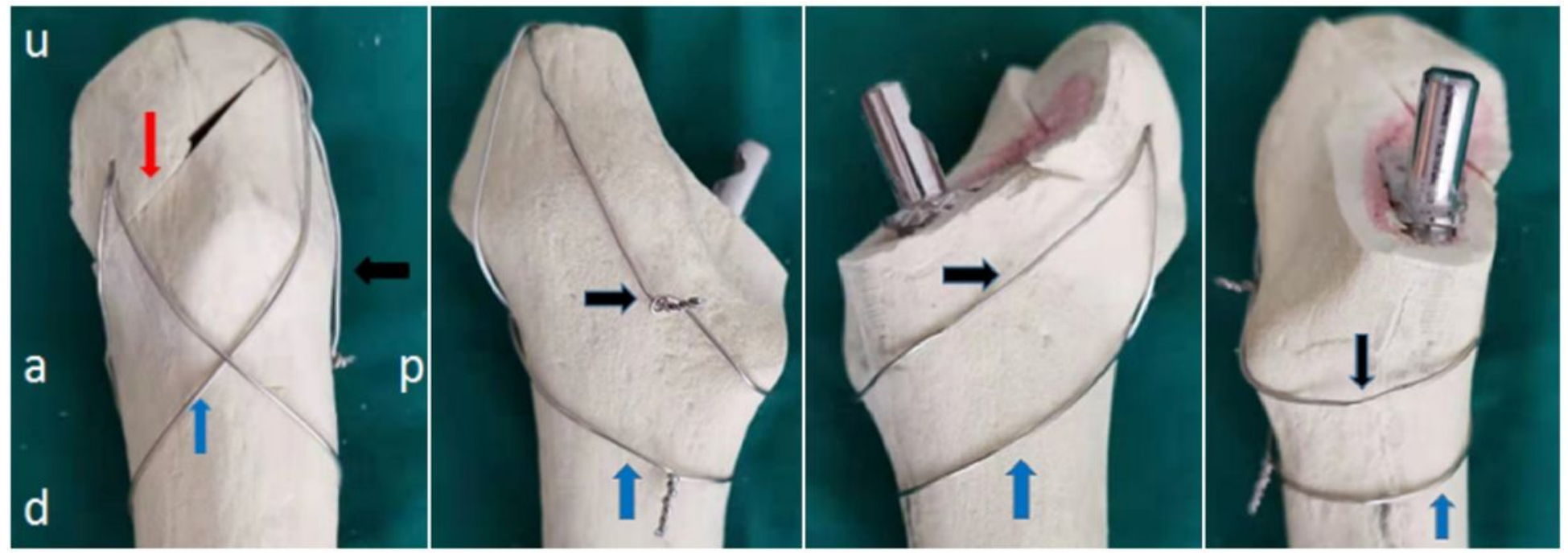

\section{Figure 4}

Type B2 fractures Type B2 fractures run obliquely from the tip of the greater trochanter to the base on the opposite side of the lesser trochanter. The red arrow represents the fracture line. A steel wire (blue arrow) perforates the tip of the trochanter and is shaped into a figure eight to fix it on the side of the lesser trochanter. The wire is tightened. A second steel wire (black arrow) also penetrates into the tip of the rotor, is fixed in a ring on the side of the lesser trochanter, and is tightened as a ventral compression fixation wire. The tightening position of the wire is behind the fracture line of the greater trochanter, and both wires bypass the lesser trochanter, with $\mathrm{u}, \mathrm{d}, \mathrm{a}$, and $\mathrm{p}$ denoting the up, down, anterior, and posterior anatomic directions, respectively. The steel wire is $2 \mathrm{~mm}$ in diameter (Resorba medical $\mathrm{GmbH}$, Germany). 


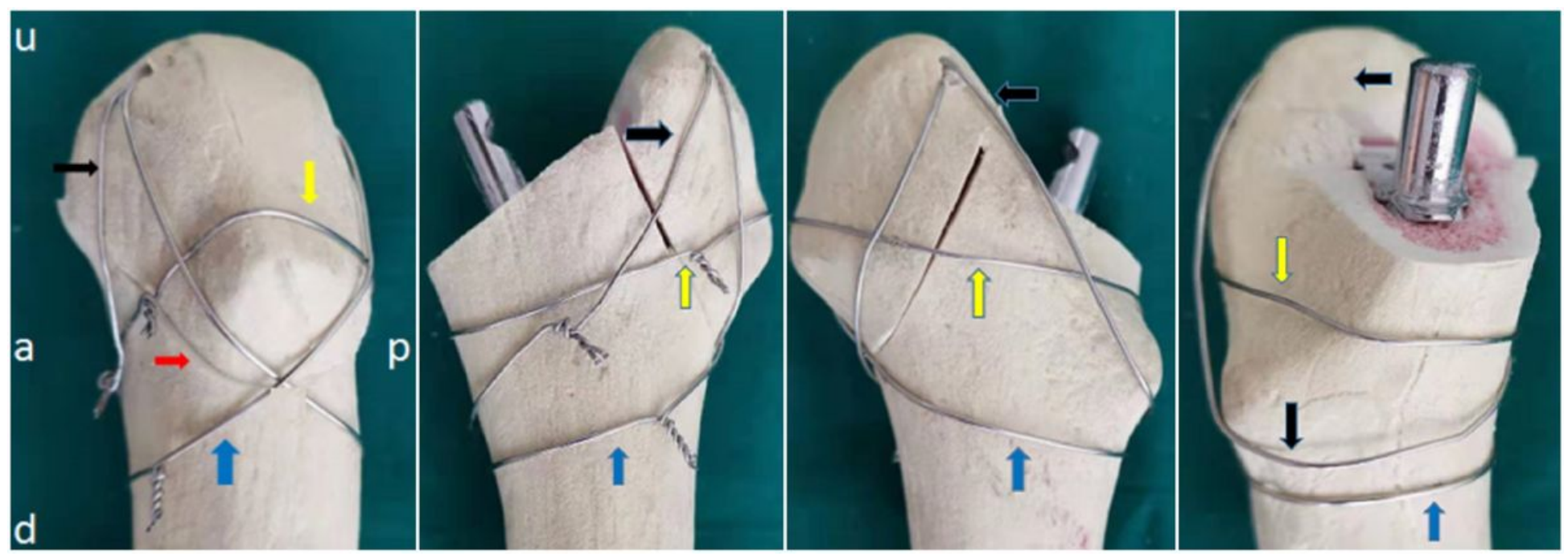

\section{Figure 5}

Type $\mathrm{C}$ fractures Type $\mathrm{C}$ fractures run across the greater trochanter and involve the lower plane of the trochanter. The red arrow represents the fracture line. A steel wire (blue arrow) perforates the tip of the trochanter and is shaped into a figure eight and fixed on the opposite side of the lesser trochanter. A second steel wire (yellow arrow) is tightened. The fracture block is fixed in a circular direction perpendicular to the fracture line. A third steel wire (black arrow) penetrates the trochanter's apex hole and is fixed on the opposite side of the lesser trochanter as a ventral compression fixation wire. The lower part is fixed, and the second steel wire (yellow arrow) is fixed above or below the lesser trochanter according to the direction of the fracture line, with $u, d, a$, and $p$ denoting the upward, downward, anterior, and posterior anatomic directions, respectively. The steel wire is $2 \mathrm{~mm}$ in diameter (Resorba medical $\mathrm{GmbH}$, Germany). 


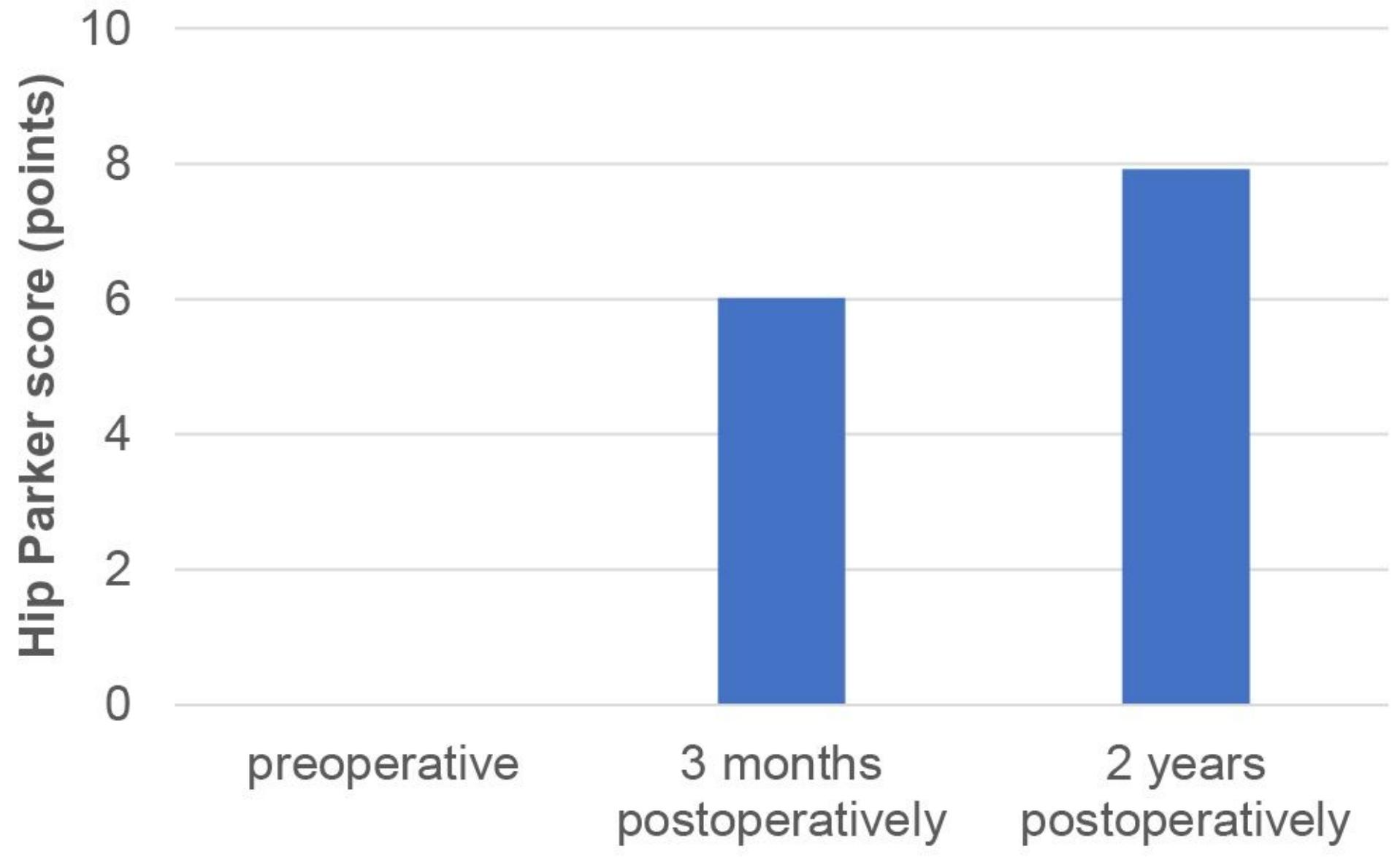

Figure 6

Parker activity scores The mean Parker activity scores of the 38 patients included in this study are shown preoperatively, three months postoperatively, and two years postoperatively. Hip joint activity improved after surgery. 
10

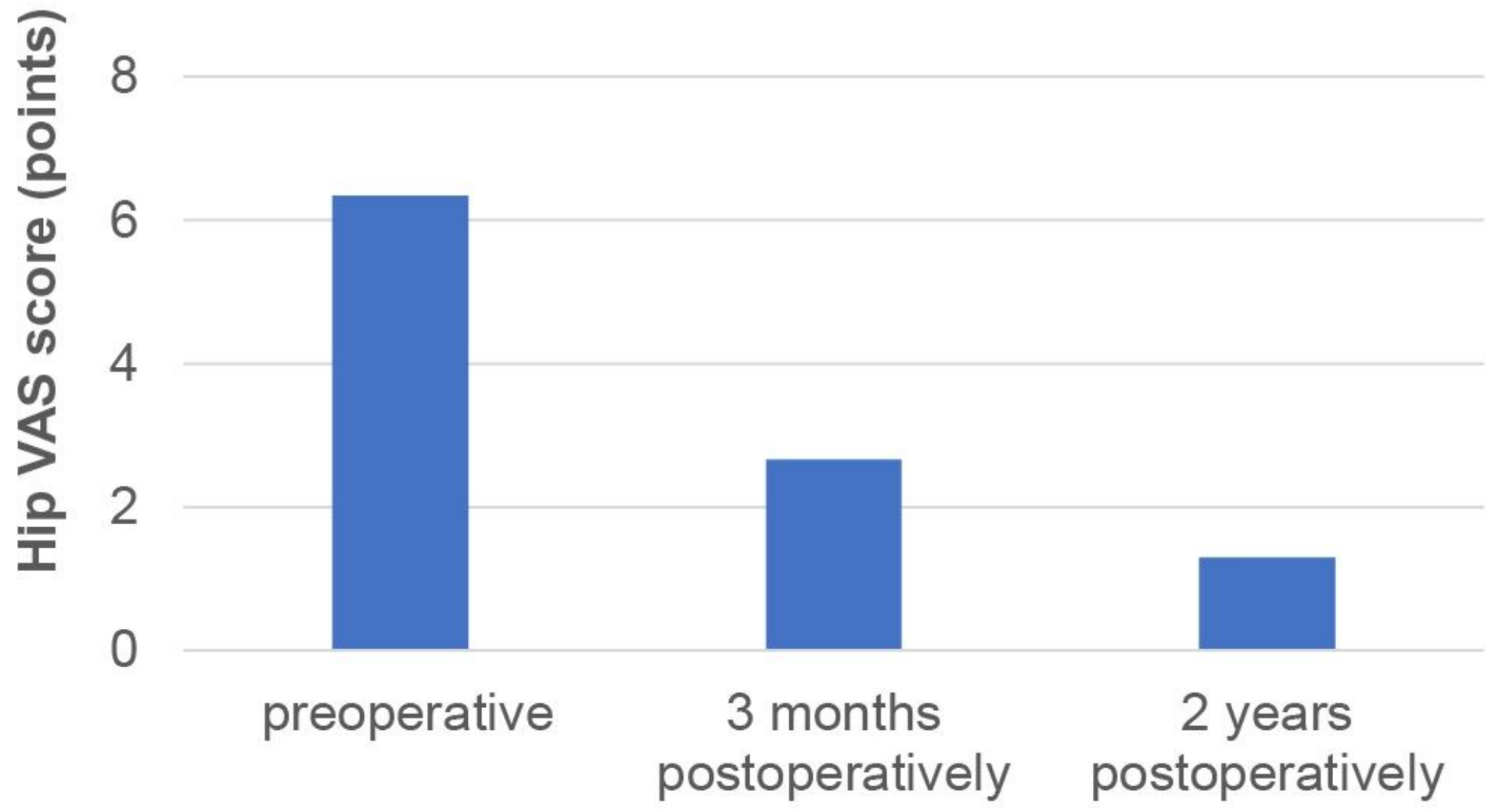

Figure 7

Hip joint visual analogue scale pain scores The visual analogue scale pain scores of 38 patients are shown preoperatively, three months postoperatively, and two years postoperatively. Pain scores improved postoperatively. 


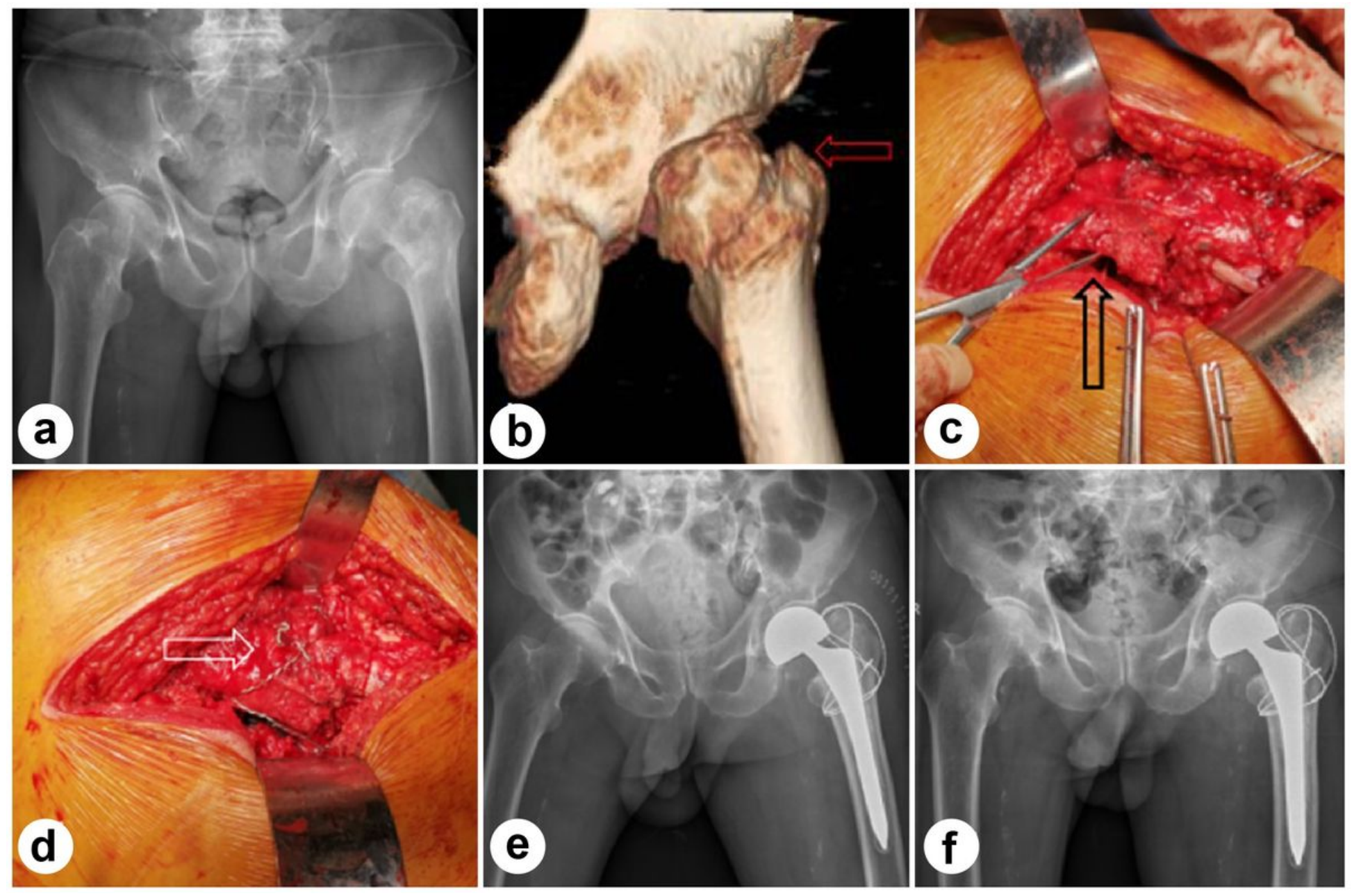

\section{Figure 8}

Typical case 1 a: The preoperative radiograph of a 76-year-old female with a left intertrochanteric fracture with obvious displacement is shown. b. A three-dimensional CT of the left hip joint shows that the fracture in the greater trochanter runs obliquely from the upper, anterior region to the lower, posterior region (red arrow). This fracture is classified as a type B2 fracture. c. The position of the displaced greater trochanter during the operation is indicated by the black arrow. $d$. The wire tension band is fixed by ventral compression wiring (white arrow) after the greater trochanter fracture is fixed. Overall, two steel wires are used: a ventral compression ring steel wire and a fixed steel wire in the shape of a figure eight. e. A pelvic radiograph obtained immediately postoperatively shows that the greater trochanter fracture is reduced and fixed well. f. A pelvic radiograph obtained during follow-up shows that the fracture of the greater trochanter is healed well and that the steel wire is not broken or displaced. 


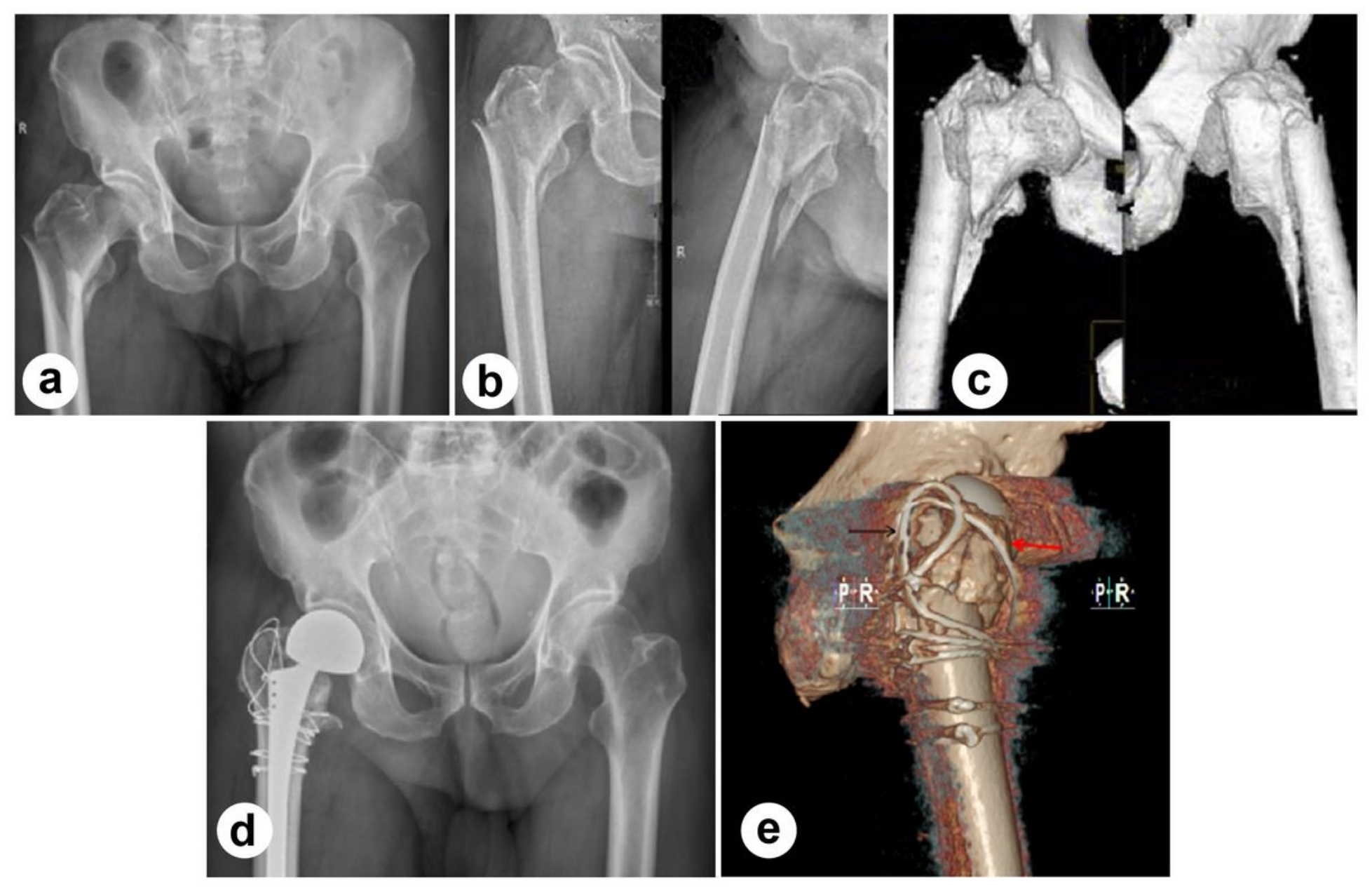

\section{Figure 9}

Typical case $2 \mathrm{a}, \mathrm{b}$. A preoperative frontal view of the pelvis and lateral view of the upper and middle right femur reveals a comminuted fracture of the right femoral trochanter with obvious displacement. c. A three-dimensional CT of the right hip joint indicates that the greater trochanter is fractured at the tip of the greater trochanter to below the lesser trochanter, indicating a type $C$ fracture. $d$. An anterior pelvic radiograph obtained postoperatively shows the femur that has been fixed via ventral compression wiring with a steel wire tension band. e. A three-dimensional CT reconstruction of the hip joint created postoperatively shows the five steel wires that were used in this fixation. Three of the steel wires were used mainly to fix the subtrochanteric bones. The red arrow indicates the ventral compression ring steel wire, and the black arrow is in the shape of a figure eight. 